\title{
LE SENTIMENT DE LA MORT CHEZ JULES SUPERVIELLE
}

María Muñoz Romero

Jules Supervielle aimait énormément la vie. Peu de poètes auront mieux que lui loué le simple fait d'être, aussi bien rendu grâces, dans leurs paroles et dans leurs actes, à la merveille d'exister. Mais il fait des compliments aux merveilles sans fermer les yeux sur leur contraire. S'il existe un regard sans oeillères, c'est le sien. Ainsi la vie n'était si forte et si vivante autour de Supervielle que dans la mesure, probablement, où il avait besoin de compenser cette expérience de la fragilité, de la menaçante mort qui fut la sienne.

En effet, dès son enfance, le poète a été obsédé par la mort, et toute son oeuvre en porte l'empreinte. Mais il n'y a pas de pathétique, et absolument rien de morbide, de frileux, de maladif, chez lui. Il avait été doté par la nature d'un coeur un peu capricieux, irrégulier. Mais sa poésie n'est pas du tout une poésie pathologique, une poésie de malade. Comme on fait un noeud à son mouchoir pour se faire souvenir d'un rendez-vous, Supervielle avait des noeuds à ses artères pour s'empêcher d'oublier qu'il mourrait. Mais il n'en a jamais déduit que rien ne valait la peine de rien.

Ce que nous avons assurément de plus commun avec nos confrères les hommes, c'est l'expérience de la mort des autres et le pressentiment de la nôtre. Cependant, la mort ne suffit point à fonder une attitude générale de l'humanité. La certitude d'être mortel ne suffit même pas à faire naître des sentiments analogues. La mort inévitable incite celui-ci à l'indifférence et cet autre à la passion, à la fureur de vivre; tel y voit des motifs de bonté et tel autre des raisons de méchanceté. Les hommes se perdent dans la mort, plus qu'ils ne s'y rejoignent. La mort rassemble tout ce qui a vécu, mais ne fait ressembler personne à personne.

Quant à Supervielle, la mort n'a presque jamais cessé de hanter sa poésie, presque jamais elle n'a cessé d'apparaître ou de se laisser deviner dans le corps du poème. Il sentit s'ouvrir dans la parole poétique l'issue par laquelle pouvait faire irruption jusqu'à nous le mouvement obsessionnel qui l'habitait. Pourtant, il ne croit pas au néant, et en même temps il n'a pas dans l'immortalité la sûre 
espérance que donne certaine foi. Sa démarche sera d'établir avec la mort un rapport de liberté. Il essayera d'humaniser et de maîtriser par un acte patient l'étrangeté de la mort. Et dans cet effort pour renforcer la familiarité confiante dans la mort, le poète cherchera surtout à maîtriser notre peur. Ce que nous redoutons comme énigme n'est inconnu que par l'erreur de notre crainte qui l'empêche de se faire connaître. C'est notre épouvante qui crée l'épouvantable.

\title{
1. À la frontière des vivants et des morts
}

\author{
"Pour dominer le silence assourdissant \\ Qui voudrait nous séparer, nous les \\ morts et les vivants" 1.
}

On a beaucoup parlé, à propos de Supervielle, de poésie métaphysique. C'est peut-être parce qu'il a entretenu la mort. Mandiargues va jusqu'à affirmer qu'il «n'écrivit jamais rien d'où la mort fût tout à fait absente»².

En effet, lui qui a su si bien chanter la vie, marque pour la mort une familiarité amicale dénuée de toute pose philosophique. La mort est chez lui l'un des thèmes dominants. C'est le point d'orgue de cette voix enjouée et grave qu'est la sienne. «Je suis né, a dit Supervielle dans son livre de mémoires Boire à la Source, sous les signes jumeaux du voyage et de la mort». Il avait huit mois lorsqu'il perdit, d'un coup, son père et sa mère. Il y a eu pour lui au départ cette séparation, il y a eu cette absence. C'est, nous le croyons ainsi, une première source. Elle ne finit pas de couler de ses premiers à ses derniers poèmes. Depuis, a-t-il dit, «l'idée de la mort a fermenté dans mon coeur». Ses parents, il les cherchera sans cesse. Au vrai, il ne pourra jamais croire à la mort, parce qu'il n'admettra jamais leur mort. Leur présence bougera en lui, rôdera autour de lui. Il les imaginera survivant dans quelque abîme sidéral ou marin, ou dans le fond de lui-même. Pour les retrouver, il tâtera l'espace avec des mots, comme avec des mains aveugles. Jamais l'espoir ne le quittera.

Le vide causé par leur disparition, il le sent à l'intérieur de lui-même, comme si, semblable à l'écoulement du temps, leur mort avait emporté quelque chose de son propre être, comme si l'essence de son moi était morte avec eux. Cette préoccupation se transforme bientôt en la recherche des parents, surtout de la mère. Écrivant sur cette recherche de la mère, Christian Sénéchal ajoute ceci:

«L'amour filial est tellement lié à la phase obscure de l'enfance et aux habitudes de la vie quotidienne, qu'on n'en ressent toute la force que dans l'éloignement out l'absence de la mort. Et il se manifeste alors sous forme de regrets et de gratitude. Pour celui qui n'a aucun souvenir vivant des siens il ne s'agit plus

1 «Le portrait», Gravitations, Paris, Gallimard, 1966, p. 90.

2 André Pieyre de Mandiargues, N.R.F., octobre 1960, p. 596. 
seulement d'une nostalgie de baisers, de sourires, de caresses et de consolations, mais de la recréation d'un être et de ce qui vous y rattache, de ce que, très heureusement, Jean Prévost a appelé une 'réinvention de l'amour filial'» ${ }^{3}$.

Or, la recherche de la mère qui préoccupe non seulement l'adolescent Supervielle, mais aussi l'homme mûr, est évidemment tout cela. Plus encore est elle, nous paraît-il, une tentative pour retrouver son propre moi, le point fixe de l'être, la source originelle.

Le tout premier poème que nous avons du poète est à la mémoire des parents:

«Il est deux êtres chers, deux êtres que j'adore,

Mais je ne les ai jamais vus,

Je les cherchais longtemps et je les cherche encore.

Ils ne sont plus... ils ne sont plus» ${ }^{4}$.

Afin d'opérer la recréation, Supervielle se tourne vers les objets qui leur appartenaient ou qui les rappellent, tels les bijoux, une montre, un portrait dont il est si souvent question dans ses poèmes. L'objet sert souvent de lien entre le poète et les disparus. Il s'agit d'une mémoire toute primitive et extérieure, le souvenir n'étant plus dans le sujet mais projeté au-dehors dans le monde des objets; d'où la complicité inattendue entre les bijoux de la mère et le poète qui, pourtant, ne les avait jamais vus auparavant. Le souvenir de la mère étant en eux, la communication est directe:

«Immobiles depuis vingt ans,

Les pauvres joyaux semblaient vivre

Comme les pages d'un vieux livre

Sous mes doigts craintifs et tremblants.

Et sans les avoir jamais vus,

Un à un, je les reconnus» ${ }^{5}$.

De même, la portrait des parents semble, sous la force de la volonté de reconstitution, s'imprégner de vie. Si forte est cette impression qu'elle frise l'hallucination auditive:

«Soudain, vos voix d'antan sur vos portraits écloses

Vinrent jusqu'à moi doucement,

Elles disaient tout bas d'imperceptibles choses

Dans un berceur chuchotement» ${ }^{6}$.

C'est à cette même recherche que nous assistons dans «Le portrait», chefd'oeuvre qui ouvre le recueil intitulé Gravitations, et qui constitue la première tentative véritable pour surmonter la séparation des vivants et des morts. Pour la

3 Christian Sénéchal, Jules Supervielle, poète de l'univers intérieur, Paris, Flory, 1939, pp. $170-171$.

4 «Hymne du jeune orphelin», Brumes du passé (premier poème du recueil), 1900.

5 «Les bijoux», Comme des voiliers, Paris, coll. de la Poétique, 1910, pp. 51-52.

6 «In memoriam: Les Portraits», Ibid. 
première fois, le poète, au lieu de constater simplement le vide qu'il ressent, nous montre les modalités de sa recherche. Il s'agit d'un chant, tour à tour rude et doux, à sa morte de vingt-huit ans qui lui a tant laissé d'elle:

«Peut-être reste-t-il encore

Un ongle de tes mains parmi les ongles de mes mains,

Un de tes cils mêlé aux miens;

Un de tes battements s'égare-t-il parmi les battements de mon coeur,

Je le reconnais entre tous

Et je sais le retenir».

En forçant la mémoire, il veut revivre les huit mois qu'il a vécus avec elle: qu'il n'y ait donc plus d'obstacle entre lui et le portrait:

«Mère, je sais très mal comme l'on cherche les morts,

Tant de gestes nous séparent, tant de lévriers cruels!»

Penché sur la photographie, le poète se met à rêver, s'imaginant vivre avec sa mère, montant et descendant les montagnes de la vie. Mais ces images floues et irréelles manquent de vérité. Sa mère et lui ressemblent à des «blessés sans mains» qui, coupés de la vraie vie, ne peuvent plus entrer en contact avec le monde, ne peuvent plus rien saisir.

À partir de ce moment, l'expérience du poète prend la dimension verticale. Si pour dominer le silence bruyant des morts, il monte sur des «toits glissants», c'est qu'il veut se rapprocher du ciel, séjour des morts:

«Je te parle durement, ma mère;

Je parle durement aux morts parce qu'il faut leur parler dur,

Debout sur des toits glissants,

Les deux mains en porte-voix et sur un ton courroucé,

Pour dominer le silence assourdissant

Qui voudrait nous séparer, nous les morts et les vivants».

Après ce bref surgissement vertical, le mouvement s'inverse et le poète descend littéralement dans le passé. Ce sont les bijoux de sa mère qui déclenchent cette nouvelle rêverie, cette nouvelle exploration de la profondeur. Enfermés dans le coffret, ces bijoux ne pourront-ils pas lui rendre sa mère? Avec l'aide de cette mémoire toute primitive dont nous venons de parler, il essaie en vain de pénétrer la nuit profonde du coffret. Écoutons ce que dit Bachelard de l'image du coffret et des bijoux, archétype de l'imagination humaine:

«Si dans le coffret il y a des bijoux et des pierres, c'est un passé, un long passé, un passé qui traverse les générations que le poète va romancer» ${ }^{7}$.

Mais l'effort sera vain:

7 Gaston Bachelard, La poétique de l'espace, París, P.U.F., 1958, pp. 87-88. 
«J'ai de toi quelques bijoux comme des fragments de l'hiver

Qui descendent les rivières,

Ce bracelet fut de toi qui brille en la nuit d'un coffre

En cette nuit écrasée où le croissant de la lune

Tente en vain de se lever

Et recommence toujours, prisonnier de l'impossible».

Dans le même chapitre du même ouvrage, Bachelard, en parlant du coffret, ajoute ce commentaire:

«Le travail du secret va sans fin de l'être qui cache à l'être qui se cache. Le coffret est un cachot d'objets. Et voici que le rêveur se sent dans le cachot de son secret. On voudrait ouvrir et l'on voudrait s'ouvrir» ${ }^{8}$.

Alors, selon cette interprétation, le poète ne chercherait pas seulement sa mère au fond de ce coffret, mais aussi bien son moi profond. Il espérerait voir resurgir de la nuit profonde de ce coffre, au-delà l'océan des âges, son moi originel, absolu.

Mais le poème se termine par une défaite, et si nous avons raison de soutenir que cette recherche est doublée d'une recherche du moi, dire alors que le poète s'est trouvé est manifestement faux. Ce qu'il retrouve c'est la conscience de ne plus être «un»: le vrai moi reste toujours ailleurs.

En ce qui concerne la recherche de la mère, le poète se rend de plus en plus compte de l'absurdité de sa tentative, La mère est bien morte, la photographie n'est qu'une photographie. Dans la dernière strophe c'est le retour à la raison: la rêverie du poète qui revient maintenant à un monde mort, figé dans l'immobilité, n'était que mensonge:

«Cigales de cuivre, lions de bronze, vipères d'argile,

C'est ici que rien ne respire!

Le souffle de mon mensonge

Est seul à vivre alentour».

Ces vers expriment en effet l'échec de l'expérience onirique. Toutes les issues sont bouchées. Mais le poète, malgré tout, se sent plus près des morts, non pour les avoir ressuscités mais parce qu'il sait que la mort est déjà en lui et que le temps avec son écoulement irrésistible continuera à user son moi jusqu'à l'effacement dernier. Cette mort, déjà en lui, et le temps sont réunis dans l'image du pouls:

«Et voici à mon poignet

Le pouls minéral des morts».

On peut rapprocher «Le Portrait» d'autres pièces appartenant au même recueil inspirées par l'image maternelle, telle que «La Belle Morte» ou «Les yeux de la morte». Dans tous ces poèmes, Supervielle exprime le même désir de redonner vie à la morte, de construire de l'existence autour de ce néant. Il se refuse à accepter la défaite.

3 Ibid., pp. 90-91. 
Le tourment de n'avoir pas connu ses parents, sa mère en particulier, est sans doute à l'origine du sentiment de fraternité qu'il ressent pour les morts, ainsi qu'à l'origine du mythe de leur survie.

Supervielle se trouve ainsi sur la frontière des vivants et du monde magique des morts. Par delà de la vie, il sait voir le monde des morts qui continuent de vivre dans l'esprit de ceux qui ne les oublient pas. Un moyen de tromper la mort serait donc d'établir un contact avec les morts qui, dans leur absence, continuent à être présents ${ }^{9}$. Ainsi, dans «Observatoire», le poète séparé de sa mère par le long fleuve de la mort, inaccessible pour lui et qu'elle a déjà traversé, trouve finalement le rapprochement cherché: doués d'ubiquité, les morts viennent se mêler aux pensées des vivants; leur silence même suffit pour que ceux-ci les comprennent:
«Le plus large fleuve du monde
Me cachait vos yeux et vos bras
Mon coeur devint sans le savoir
Une île sous les eaux profondes,
Elle n'osait se laisser voir.
Plus tard, vous étiez si près
Que j'entendais votre silence» ${ }^{10}$.

Et ce silence qui se forme entre eux, lui aussi est fécond. Leurs âmes y sont plus proches. Plongés dans le doute, les hésitations et la timidité, les trois derniers vers de ce poème («Observatoire») nient, malgré tout, la frontière qui sépare les morts des vivants:

«Mais n'êtes-vous pas devenue

L'astronome d'un autre monde

Qui me suit de sa longue-vue?»

Ainsi donc, même si nous ne pouvons pas les voir, les morts nous suivent des yeux partout où nous allons, leur regard traversant l'éther d'au-delà de millions d'années-lumière. Cela nous rappelle le conte de Supervielle intitulé $\mathrm{La}$ femme retrouvée ${ }^{11}$, où le protagoniste, à travers d'énormes télescopes installés dans le ciel, pouvait observer sa femme sur terre et suivre chacun de ses mouvements.

Dans «Projection», la présence des morts est rendue toute proche et certaine. Le poète y entend le «piétinement / De la vie et de la mort qui troquent leurs prisonniers» dans un «cimetière aérien» ${ }^{12}$.

En effet, la relation des morts et des vivants est au coeur même de son oeuvre: «J'ai toujours essayé, dit-il à Nadal, de dominer le silence insupportable des

9 Dans une note publiée par la N.R.F. (oct. 1960, p. 768), Supervielle écrit. «Un mort, même enterré, est terriblement présent. Par l'intensité même de ma fausse présence, par mon mutisme redoublé, je serai infiniment plus présent que si je n'étais pas mort».

10 «Observatoire», Gravitations, p. 138.

11 La femme retrouvée dans L'Arche de Noé, Paris, Gallimard, 1938, pp. 129-185.

12 «Projection», Gravitations, p. 141. 
morts» ${ }^{13}$. Le poète se sent chargé d'une mission. Il doit aux morts fidélité et affection. Il lui appartient de nourrir leur souvenir, de s'approcher d'eux, de leur parler. Cette démarche ne va pas sans scrupules: il sent qu'il peut les blesser par son poids de vivant. Elle ne va pas sans déception: il sait que la frontière entre eux et lui ne sera pas franchie tant qu'il vivra. Il persiste, malgré tout, à leur tendre la main et imagine, de l'autre côté, d'autres mains tendues vers la sienne, une fascination de la vie pareille à la fascination de la mort à laquelle il s'abandonne. Il rêve ainsi à de multiples formes de survie, heureuse ou malheureuse, toujours en proie au même désir de naître.

Sa poésie conteste la victoire prétendue de la mort. Il abolit, au monde de ses fables, la distance qui sépare les deux rives du fleuve infernal. Il va et vient de l'une à l'autre. Il assiste les âmes «veuves» qui ne savent plus à quels corps se vouer et qui sollicitent du poète la faveur de «s'appuyer un peu sur ses pensées». Ici-bas, il se demande s'il est bien présent. Il ne parvient pas à devenir un «professionnel de l'existence». Ainsi se conduit Supervielle, le «hors-venu». Nulle situation ne le fixe. Nul état ne le définit. Il n'est vraiment ni d'ici ni d'ailleurs. Il traverse la vie et la mort sans jeter l'ancre.

La vie et la mort s'interpénètrent si merveilleusement qu'il devient impossible de savoir où finit l'une, où commence l'autre:

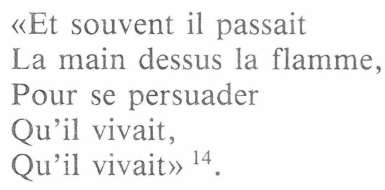

L'univers du poète en acquiert ainsi sa forme. C'est l'univers d'un PASSAGE: le passage du visible à l'invisible. Les deux plans sont liés; ils se suivent comme l'ombre du corps suit le corps. Vivants et morts sont unis:

«Mais en nous rien n'est plus vrai

Que ce froid qui nous ressemble.

Nous ne sommes séparés

Que par le frisson d'un tremble» ${ }^{15}$.

Et plus loin:

«Nous avons partie liée

Tel l'époux et l'épousée

Quand il souffle la bougie

Pour la longueur de la nuit» ${ }^{16}$.

Un univers de passage, où rien ne disparaît, ne cesse d'être. Où rien n'est jamais perdu, où demeure ce qui fut. En bref, un univers peuplé, foisonnant, un univers de présences:

13 «Conversation Octave Nadal-Supervielle», N.R.F., oct. 1960, p. 618.

14 «Pointe de flamme», Gravitations, p. 165.

15 «Oloron-Sainte-Marie», Le Forçat innocent, Paris, Gallimard, 1980, p. 50.

16 Ibid., p. 52. 


\begin{abstract}
«Dans la forêt sans heures
On abat un grand arbre

Un vide vertical

Tremble en forme de fût

Près du tronc étendu» 17 .
\end{abstract}

On passe dans cet univers du visible à l'invisible, mais non pas de manière abrupte. Ce monde fourmille de connivences, on s'y donne des rendez-vous, une mystérieuse galantérie s'y déploie, d'étranges unions s'y consomment. Et de la vie à la mort s'opèrent, chemin faisant, des métempsychoses, des métamorphoses. La mort n'est pas disparition, mais changement d'état, migration d'une forme à une autre forme. On ne meurt pas, on mue.

Une telle concentration de l'esprit et des sens pour établir le contact avec les morts, allait du moins créer une intimité plus forte que l'effroi et la douleur. Tout le cycle du «Miroir des morts» (Gravitations) est proprement de la métapsychique sans ectoplasmes, du spiritisme sans tables tournantes. Cheveux, lèvres et carnation gardent dans l'espace une sorte d'existence atténuée:

«Tes cheveux et tes lèvres

Et ta carnation

Sont devenus de l'air

Qui cherche une saison» ${ }^{18}$.

Comme dans les croyances animistes des primitifs, les morts nous entourent invisibles. Par contre, le vivant a besoin d'une «pointe de flamme» pour se persuader qu'il vit. Vivants et morts se rapprochent donc et se mêlent. Des voix emplissent l'espace pleines de souvenirs et de désirs humains.

Alors qu'il aime à se représenter les morts comme vivants encore, et ne se décidant pas à mourir tout à fait, il a le don de sentir les secrètes intrusions de la mort dans la vie.

La tristesse première s'est muée en gravité confiante. La mort est, dans la vie, une communion avec les âmes désincarnées. Elle «cesse d'être néant ou audelà, pour former une atmosphère» ${ }^{19}$. Le rôle du poète sera de «rassembler les morts qu'une rumeur effarouche», de porter à ceux qui «rôdent autour de notre position» le message de la vie. Il a eu toujours l'idée qu'on pourrait faire quelque chose pour eux... Il fallait les sauver, les ressusciter par la force du souvenir humain.

En effet, derrière ces rêveries de poète nourries par de naïves histoires de revenants, on devine toute une expérience de la vie intérieure. C'est un fait qu'après leur mort physique, les défunts subissent dans les mémoires une seconde mort,

17 Ibid., p. 111.

18 «La Belle Morte», Gravitations, p. 167.

19 Christian Sénéchal, op. cit., p. 102. 
spirituelle. L'oubli les détruit plus sûrement que les vers du tombeau. On éprouve alors comme un remords d'avoir laissé mourir ceux qui auraient pu vivre en nous, on se sent coupable, on s'incline à penser qu'ils nous supplient de leur rendre quelque réalité en ranimant leur image dans notre esprit.

Quand Supervielle les imagine assiégeant les vivants, il transpose cette vieille idée que nous leur devons un culte, un culte qui est beaucoup plus qu'un hommage: une façon de les ramener parmi nous et de leur rendre la lumière dont ils ont été frustrés.

$\mathrm{Au}$ reste, le revenant qui le hante le plus est le mourant qui agonise en lui à chaque minute, son «double», son moi «posthume». Il est au fond de lui même, s'accrochant à un corps qui va lui échapper:

«Un sourine préalable

Pour le mort que nous serons,

À ce mort que nous serons

Qui n'aura qu'un peu de terre,

Maintenant que par avance

En nous il peut en jouir...» ${ }^{20}$.

Enfin, il se pourrait bien que les fantômes supervilliens ne traduisent pas seulement, de façon purement symbolique, une expérience de la mort intérieure, mais qu'ils aient quelque réalité objective aux yeux du poète. Pour qu'ils soient si nombreux dans ses livres, il faut bien qu'il leur accorde une part d'existence. Il le fait, sinon avec la raison, du moins avec son imagination qui refuse de considérer la mort comme une fin absolue.

\section{Peur de la mort, acceptation de la mort}

\section{«Notre crainte de mourir Notre douceur de mourir» ${ }^{21}$}

Nous avons déjà vu comment le thème de la mort est un des thèmes majeurs de l'oeuvre supervillienne. Thème nullement nouveau, certes, et que sans son actualité, toujours pressante, nous serions tentés de traiter moins d'éternel que de sempiternel.

Mais, ce thème, le poète uruguayen l'a rajeuni. Ni stoïcisme guindé, chez lui, ni facile résignation chrétienne, ni révolte romantique, mais une acceptation lucide; quelque chose comme la conscience, non de la destructibilité, mais de la solubilité de l'être; une sorte de notion d'éternité consistant, d'une part, dans le fait irrévocable d'avoir «été», de l'autre, dans le retour, non au néant, mais aux sources de l'énergie, partant à de nouvelles possibilités.

20 «Offrande», Gravitations, p. 172.

21 Ibid. 
Ce serait difficile de démêler ce qu'il peut y avoir, dans une telle conception, d'antique et de moderne, de païen et de chrétien. Contentons-nous de dire que l'image que nous offre Jules Supervielle de la survie a la grâce pure, la tendre sérénité de l'art grec. Qui pourra, en effet, lire ce court poème sans évoquer ces stèles funéraires où le mort est représenté encore installé dans la vie, dans ses attitudes familières, avec seulement, pour nous avertir, un «air d'absence» plus émouvant que toutes les contorsions?:

«Tout le long de sa vie

Il avait aimé à lire

Avec une bougie

Et souvent il passait

La main dessus la flamme

Pour se persuader

Qu'il vivait,

Qu'il vivait.

Depuis le jour de sa mort

Il tient à côté de lui

Une bougie allumée

Mais garde les mains cachées» ${ }^{22}$.

Il nous ramène ainsi de son imagination des défunts à sa propre relation avec l'idée de la disparition finale, et en évoquant sa ressemblance anticipée avec les morts, il nous fait comprendre que par cette idée-là une réconciliation est peutêtre possible. De son vivant, il essaie de se faire écolier de la mort, et d'acquérir cette «science» qu'il a parfois prêtée à certaines de ses images de défunts. Le Dieu de La Fable du Monde a dit aux hommes avec une mélancolique bonhomie: «chacun de vous sait faire un mort sans avoir eu besoin d'apprendre» ${ }^{23}$.

Comme son ami Rilke, Supervielle pense que chacun contient sa mort comme le fruit son noyau. On a bien sa mort, et cette conscience nous donne une dignité, une silencieuse fierté. La mort mûrit au sein de nous-mêmes, elle est le fruit que nous devons porter et nourrir. Il ne doit pas seulement y avoir mort pour nous au tout dernier moment, mais mort dès que nous vivons et dans l'intimité et la profondeur de la vie. La mort n'est donc pas un terme vers lequel l'homme tendrait. Elle est dans la vie même. Les os constituent la marque matérielle la plus évidente de cette présence. Chez Supervielle, le squelette représente la mort incrustée en nous. Dans le poème du Forçat innocent, "Oloron-Sainte-Marie», le poète dialogue avec ses os, leur demande de patienter, de prendre goût à la chair et à la vie.

La mort devient ainsi partie de la vie. C'est un danger auquel il faut apprendre à s'habituer. Elle vit de nos vies, dans le plus intérieur de nous. Elle est faite de nous et pour nous. En définitive, nous engendrons notre mort, ou bien, comme dit Rilke, «nous mettons au monde l'enfant mort-né de notre mort».

22 «Pointe de flamme», Ibid., p. 165.

23 «Tristesse de Dieu», La Fable du Monde, París, N.R.F., 1938. 
Il faut que notre mort nous devienne toujours plus intérieure: qu'elle soit comme notre forme invisible, notre geste, le silence de notre secret le plus caché. Nous avons tout à faire pour la «faire», elle doit être notre oeuvre. Nous devons être les figurateurs et les poètes de notre mort.

«L'on ne peut écrire, affirme Maurice Blanchot, que si l'on reste maître de soi devant la mort, si l'on a établi avec elle des rapports de souveraineté. (...) L'art est maîtrise du moment suprême, suprême maîtrise» ${ }^{24}$.

L'écrivain est alors celui qui tient son pouvoir d'écrire d'une relation anticipée avec la mort.

«Bien mourir, continue Blanchot, signifie mourir dans la convenance, conformément à soi, dans le respect des vivants. Bien mourir est mourir dans sa propre vie, tourné vers elle et détourné de la mort, et cette bonne mort indique plus de politesse pour le monde que d'égards pour la profondeur de l'abîme» ${ }^{25}$.

Ainsi, pour Supervielle, la mort devient une soeur. La crainte s'abolit. Mais non la tristesse: la tristesse de changer de forme, la tristesse devant les mille fuites du monde, devant l'impossibilité de «saisir». Il s'arrache cependant à l'angoisse, parce qu'il croit que, dans la mort, la vie continue quand même, si atténuée soitelle. Il imagine la vie future comme une transmutation de la vie présente, non comme son abolition totale.

Il lui faut évidemment une dose singulière de résignation pour se contenter d'une existence si réduite! Mais il finira pour la trouver en lui. Après avoir senti avec tristesse que tout en lui menaçait de se disloquer, après avoir redouté sa fin, il parvint à la considérer avec une sorte de tendresse.

En effet, sans gestes, sans malédictions, une âme qui semblait vouée au désespoir, conquiert peu à peu un calme qui n'est pas lâche oubli de la menace, et, ayant apprivoisé la mort sans la priver de son mystère, elle va se risquer à explorer un coeur qui sentira un jour les glaces éternelles. La mort est entrée dans la société des «amis inconnus». Par sa présence au coeur de l'homme, elle rend les spectacles de la terre et du ciel plus émouvants de fragilité et plus lourds de sens. Peuplant l'espace de ses voix, elle prépare ceux qui prêtent l'oreille à leurs appels.

Il ne s'agit pas non plus de mourir content. Cela exprimerait le mécontentement de la vie. S'il se résigne à la mort, ce n'est pas sans se dire qu'il n'est pas fait pour elle, qu'elle lui «va» mal:

«Il faudra bien pourtant qu'on m'empaquette

Et me laisser ravir sans lâcheté,

Colis moins fait pour vous, Éternité,

Qu'un frais panier de claires violettes» ${ }^{26}$.

Bien qu'il ne soit séparé des morts que «par le frisson d'un tremble», c'est encore vers la vie qu'il se tourne, vers elle qu'il remonte, car la vie triomphe

24 Maurice Blanchot, L'espace littéraire, París, Gallimard, 1955, p. 107.

25 Ibid., p. 121.

26 «Voeu», Gravitations, p. 173. 
malgré tout. Tout en attendant la mort et en se préparant pour elle, Supervielle s'affirme encore trop vert pour mourir:

«Rien ne consent à mourir

De ce qui connut le vivre» ${ }^{27}$.

Après avoir longtemps voyagé dans et hors de ce monde, cet ami des morts reconnaît que tout est vie dans ses vers.

\title{
3. La longue plainte des morts. Le dualisme âme-corps
}

\author{
«Lorsque nous serons morts nous \\ parlerons de vie» ${ }^{28}$.
}

Sans savoir pourquoi, ceux qui nous furent chers ne seront plus un jour pour nous que «du silence». Le poète entend s'élever de ce mutisme une plainte qui n'est autre, en somme que la sienne: plainte du vide qu'ils laissent en nous, et plainte anticipative pour soi-même. L'union de ces deux peines, avec primauté de la seconde, trouve sa forme indirecte dans la longue élégie éparse et toujours reprise que Supervielle croit entendre murmurer par les âmes dépouillées de leur vie.

En effet, cette persistance par-delà la mort des voix qui continuent à appeler, à dialoguer avec le poète, fournit un des motifs les plus obsédants de l'oeuvre supervillienne:

«Le monde est plein de voix qui perdirent visage

Et tournent nuit et jour pour en demander un» ${ }^{29}$.

La mort, ce sont d'abord les morts et nous ne pouvons les oublier, parce qu'ils nous hantent, parce qu'ils nous habitent, parce qu'ils ne cessent de solliciter notre écoute. C'est qu'ils savent bien que le peu d'existence qu'ils possèdent dépend de notre acceptation. Ils attendent des vivants le contraire d'un ensevelissement: une reconnaissance de leur existence.

Considérons donc à présent de plus près et en elle-même cette légende des défunts. De quelle condition la fiction sensible du poète les pourvoit-elle?

Avant tout, elle les voit attachés à la Terre. Spiritisme? Résidu des vieilles histoires de fantômes? Nous ne le croyons pas, car les morts de Supervielle ne sont pas effrayants. Ils ne ressemblent pas aux fantômes des histoires anglaises. Ils sont des fantômes «vrais», c'est-à-dire, immatériels. Ils ne sont chargés ni de nous effrayer, ni de nous avertir. Les apparitions de Supervielle sont tendres et pitoyables. Elles n'en savent pas sur nous davantage que nous-mêmes. Elles ne

27 «Souffle», Ibid., p. 136.

28 Vers de Tristan l'Hermite, épigraphe de Gravitations.

29 Les amis inconnus, París, Gallimard, 1980, p. 137. 
possèdent pas les secrets qui nous échappent, ni les réponses qui nous fuient. Leur présence timide n'annonce que notre future absence.

Les morts de Supervielle sont des morts protestataires qui n'admettent pas leur sort, et qui se consolent en rêvant à la vie:

«Des images de rivières,

De torrents pleins de remords

Croient rouler une eau fidèle

Où se voient vivants les morts» ${ }^{30}$.

Les simulacres de vie se multiplient en vaines preuves. C'est le royaume du «virtuel» qui s'efforce de devenir le «réel». Effort faible et fragile qui ne triomphera jamais. Toutes ces prétentions dérisoires se résument dans le geste dément de l'âme qui prend pour un arbre plein de fruits un simple «mouvement du ciel»:

«L'âme folle d'irréel

Joue avec l'aube et la brise

Pensant cueillir des cerises

Dans un mouvement du ciel» ${ }^{31}$.

Elle se donne donc l'illusion de pouvoir tirer de l'invisible et de l'intangible (l'aube et la brise) du visible et du tangible (des cerises). Les cerises représentent la terre, le tangible au milieu du flou et du mouvant, et, enfin, la nostalgie du limité.

Donc, notre poète, losqu'il essaie d'imaginer l'existence des morts, ne conçoit qu'un éternel regret de la terre. Les voici se pressant dans l'antichambre de notre monde:

«On voit les morts de l'espace

Se rassembler dans les airs

Pour commenter à voix basse

Le passage de la Terre...» ${ }^{32}$

C'est que, comme le dit «Alarme», «Tout ce qui mourut sur terre / Rôde humant de loin la vie» ${ }^{33}$. Les morts ne vivent plus que de leurs souvenirs du temps qu'ils ont passé sur terre. Chacun essaie de retourner à ce qu'il fut, à ce qu'il vécut:

«Les Ombres des anciens habitants de la Terre se trouvaient réunies dans un large espace céleste; elles marchaient dans l'air comme des vivants l'eussent fait sur terre.

Et celui qui avait été un homme de la préhistoire se disait:

'Ce qu'il nous faudrait, voyez-vous, c'est une bonne caverne spacieuse, bien abritée, et quelques pierres pour faire du feu. Mais quelle misère! Rien de dur autour de nous, rien que des spectres et du vide'.

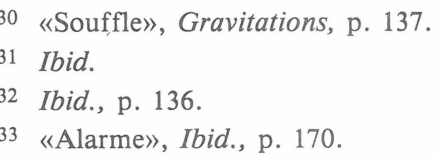


Et le père de famille des temps modernes introduisait avec précaution ce qu'il prenait pour sa clef dans le trou de sa serrure et faisait mine de fermer sa porte avec le plus grand soin.(...)

Le lendemain il faisait comme si sa barbe avait poussé durant la nuit et se savonnait longuement avec un blaireau de brouillard.(...)

Tout ce qu'on faisait sur terre se reflétait dans cette partie du ciel ...» ${ }^{34}$.

Come devant une glace, ils vivent le reflet de leur existence perdue:

«Le mort vient de dérober

Un long miroir à la vie

Il se voit monter aux arbres,

S'étonne que les oiseaux

Dans ses mains se laissent prendre

Pour y mourir aussitôt» ${ }^{35}$.

Rien n'arrêtera le perpetuel mouvement de ces défunts vers l'existence. Tous les morts sont en proie au même désir, à la même idée fixe. Les uns sont entreprenants, comme celui du «Miroir»; les autres, comme celui de «Pointe de flamme», timides et accablés, préfèrent s'aveugler, évitent tout ce qui pourrait les assurer de la réalité de leur sort. Mais cette attention pour la Terre est générale chez tous les trépassés de Supervielle. Notre monde réel est pour eux comme un miroir dans lequel ils essaient de se fixer.

Le discours des morts ne peut donc rouler que sur les affaires terrestres, et il prend nécessairement le ton de la complainte. Ils ont perdu l'autorité de la présence. Ils regrettent le concret, son épaisseur indubitable. Il leur est refusé désormais de le toucher, ce concret. Comme on le dit dans Les boiteux du ciel, «la grande tristesse des Ombres venait surtout de ce qu'elles ne pouvaient rien saisir» ${ }^{36}$.

C'est comme si l'énergie spirituelle continuait de vivre après la mort dans le seul but de regretter la vie. Et cette «survie» aura pour siège l'immensité céleste qui constituera, non pas le paradis où l'âme, libérée des contraintes de la chair, trouverait enfin la plénitude qu'elle cherchait pendant la vie terrestre, mais, au contraire, une sorte de nécropole projetée dans l'espace, un vide rendu sensible, le tombeau de l'humanité.

En effet, le ciel est la profondeur par excellence, plus terrifiante que celle de la mer ou de la terre, parce qu'elle est infinie. On essaie cependant de vaincre cette profondeur et la sensation de vertige qu'elle provoque, et où «il n'y a pas de droite ni de gauche, d'avant ni d'après et rien que la profondeur» on substitue des rues, des avenues, des carrefours, qui, malgré tout, ne peuvent rivaliser de stabilité avec ceux de la terre:

34 Les boiteux du ciel dans L'enfant de la haute mer, Paris, Gallimard, 1978, pp. 85-86.

35 «Le miroir», Gravitations, p. 164.

36 Les boiteux du ciel dans L'enfant de la haute mer, p. 92. 
«Cimetière aérien hanté de rues transversales,

De puissantes avenues

Et de quais d'embarquement...» ${ }^{37}$.

Ainsi donc, ce n'est pas le mouvement de la vie vers la mort que veut favoriser le poète. Passager du «paquebot Terre» dont «les hublots» laissent passer les «oiseaux familiers», il s'émerveille devant ce spectable et ne veut pas aller dérober je ne sais quel absolu qui risque bien d'être une absence:

«Contentons-nous d'être un vivant un jour de plus,

D'entendre en nous ce coeur qui ne s'est pas couché

Et peine nuit et jour dans d'égales ténèbres

Pour préparer un peu de ce qu'il croit bonheur» ${ }^{38}$.

En efect, sa légende des morts, avec l'élégie qu'il leur a fait si souvent reprendre, c'était encore une forme de sa propre louange de la vie.

Dans toute l'oeuvre de Supervielle domine une conception dualiste de l'homme. Notre poète prolonge la réflexion de la tradition philosophique sur la relation de l'âme et du corps. Les problèmes de ce couple viennent de ce que le corps s'inscrit dans un temps et un espace limités. L'âme en souffre, aspire à la liberté, mais ne peut se passer du corps.

\section{A. L'entrave}

Le vivant est double, mais le corps ne peut tenir toujours l'âme étroitement enchaînée. Il existe un hiatus entre elle et lui. Ils ne vivent pas le même temps. Dans l'homme de la Pampa, l'âme de Guanamiru est «de cinquante ans plus agile que ses jambes» ${ }^{39}$.

Si notre corps est situé, l'âme est «extensible jusqu'aux étoiles». Pourquoi vivre petitement, en provinciaux du monde, quand l'univers est notre domaine! Libre et lumineuse, disposant de l'espace à sa guise et ignorant les obstacles, telle apparaît l'âme chez Supervielle:

«L'immensité est l'affaire du plein air de l'âme. Le corps, lui, ne demande pas tant d'espace. Il connaît ses limites, toute cette grandeur d'âme d'un coup lui donne le vertige. Mais pour l'âme, elle n'a rien à craindre... ${ }^{40}$.

L'âme ouvre à la personne l'accès du possible, tandis que le corps la maintient au contact du réel. Le corps décline avec les ans. L'âme conserve la vigueur de la jeunesse. Elle échappe aux sens. Elle est toujours prête à s'évader, jouissant

37 «Projection», Gravitations, p. 141.

38 «Matin», Les amis inconnus, p. 205.

39 L'homme de la Pampa, París, Gallimard, 1978, p. 11.

40 Boire à la source, París, Gallimard, 1951, p. 181. 
de sa pleine liberté, laissant le corps derrière avec son fardeau de chair et d'os. L'âme sort des maisons par la fenêtre et le corps par l'escalier. L'âme va instantanément de France en Amérique, le corps doit emprunter les lenteurs de la mer. L'âme, à la différence du corps, se joue des lois de la Nature. C'est une ombre émancipée, un moi surnaturel.

Dans la poésie de Supervielle, l'existence de l'âme, bien loin de revêtir l'aspect d'un dogme, d'une révélation religieuse, se présente comme une «donnée immédiate de la conscience». Supervielle se contente d'appeler son coeur du nom d' 'âme» chaque fois qu'il le surprend en train de vivre sur le mode imaginaire, c'est-à-dire «dans l'oubli de son corps».

L'âme «spacieuse» de Supervielle hante l'immensité des cieux et l'abîme des eaux. Elle s'y sent à l'aise. Elle s'y livre follement à son humeur vagabonde. Elle s'y déplace avec volupté. Elle élève ainsi l'homme au-dessus de l'objet matériel, qui est inerte, et de l'animal, qui ne renouvelle guère ses itinéraires. Elle n'est rien d'autre que l'inspiration qui l'entraîne partout et toujours, de la naissance à la mort.

Le corps constitue pour elle une entrave à plusiers titres: chaperon inévitable, prison, matérialité. L'âme souffre, en effet, de devoir partager toutes ces limites du corps qu'elle regarde comme un compagnon encombrant et d'essence inférieure:
«J'avais honte de mon corps
Qui se présentait partout
Avec moi, m'enveloppant
De sa chair à vêtements,
Je le trouvais si grossier
Avec les os et le sang... ${ }^{41}$

\section{B. La libération et les regrets}

Ce corps et cette âme mal soudés, la mort les sépare sans peine. Ainsi, par exemple, dans La fuite en Egypte, l'âme du boeuf, après la mort de celui-ci, jouit de sa liberté toute neuve. Finies les servitudes du corps, l'obligation de brouter et de dormir, elle fait l'expérience de la «merveilleuse légèreté» et de la totale liberté de mouvement.

Mais très vite apparaît le revers. Les qualités de l'âme deviennent défauts: la légèreté n'est qu'un aspect de l'instabilité, de l'errance, de l'inconsistance. Les défauts du corps deviennent qualités: le poids devient pondération.

Le poète avait imaginé que le terme de la migration de l'âme serait le paradis céleste. Mais l'âme de Supervielle est trop flottante, sa foi en l'éternité trop peu enracinée pour que de telles visions durent longtemps. Les minutes confiantes alternent chez lui avec la conviction que la béatitude céleste n'est pas garantie. Tout bien calculé, la vraie vie est la vie sur terre, où l'âme jouit d'un corps bien charnel, bien sain, bien équilibré. Pas de plus grand bonheur que de sentir ses

41 Les amis inconnus, p. 138. 
muscles solides, ses articulations correctement huilées, ses os attachés ensemble, son coeur battant.

Ainsi, ce pauvre corps, avec ses limites, est regretté, car c'est par lui que nous entrons en contact avec le monde et avec les autres. Les âmes des morts cherchent désespérément à reprendre corps:

«Vous appellerais-je fantômes,

Amalgames de ténèbres

À la recherche d'un corps,

D'une mince volupté...» ${ }^{42}$

Certaines vont jusqu'à emprunter le corps d'un autre être vivant: dans $L a$ fuite en Egypte ${ }^{43}$, le boeuf, pour suivre Jésus, se loge dans le corps d'une vache; dans La femme retrouvée ${ }^{44}$, l'âme du protagoniste qui veut absolument revoir sa femme, revient sur terre habiter le corps d'un chien. Supervielle rend douloureusement concrète cette évidence: la mort est privation du corps, les morts sont «de grands mutilés de tout leur corps» ${ }^{45}$.

La mort sépare l'âme du corps qui la rendait «glissante et lourde». Redoutable, tragique libération! Livrée sans merci à son démon, l'âme cesse de contrôler ses écarts. Elle a perdu, avec le corps, sa boussole, son frein. «Veuve» du corps, elle erre à l'aventure, elle s'emballe, elle se perd. Si l'âme dans la mort s'envole selon son habitude, son vol sera, cette fois, sans pause et sans retour. Le voyage de la mort est donc bien le dernier voyage, celui dont on ne revient jamais.

En effet, l'un des aspects les plus originaux de la poésie de Supervielle, c'est l'attention portée par le poète au corps. Il ne peut pas concevoir l'âme sans le corps. Il ne s'intéresse qu'au mélange des deux. Les fantômes qu'il évoque ont une constante obsession: chercher un corps pour pouvoir naître, pour pouvoir «connaître»:
«Regards sans iris ni racines
Rôdant dans l'espace argentin,
$O$ regards, serez-vous enfin
Retenus par une rétine?» ${ }^{46}$

C'est que, chez Supervielle, le merveilleux ne ressemble pas à la féerie que se joue un esprit désincarné. Supervielle nous invite au contraire à rentrer en notre corps, en notre sang, à coïncider avec notre dessin terrestre, à prendre conscience de nos véritables appartenances, dans un esprit de sympathie tremblante et de tragédie secrète.

Il a compris que son premier compagnon à lui était son corps aussi proche et aussi inconnu que le reste du monde, semblable au corps du monde, irrigué

42 «Projection», Gravitations, p. 141.

43 La fuite en Egypte dans L'Arche de Noé, pp. 37-57.

44 La femme retrouvée dans L'Arche de Noé, pp. 131-185.

45 Les boiteux du ciel dans L'enfant de la haute mer, p. 86.

46 «Commencements», Gravitations, p. 111. 
comme lui, comme lui voué à l'alternance de la nuit et de la lumière, comme lui soumis au flux du temps.

Il rend au corps toute son importance. «La merveille d'être un vivant!», s'écrie le poète dans Le Corps tragique ${ }^{47}$. En de tels moments, il se sent rassuré par un espace à sa dimension, par un temps sans commune mesure avec une interminable éternité.

Un attachement aussi humain au corps entraîne souvent une dévaluation de l'Esprit. La pensée, naguère réputée créatrice, apparaît aussi vide que le vent, la survie spirituelle aussi évanescente que la fumée. Cessant d'être un principe vital, l'abstraction devient le caractère essentiel du Néant. Supervielle ne situe plus alors le royaume de la mort sur la terre pesante et fortement substantielle, mais dans l'immensité vide du ciel ou les étendues glauques de la mer, dans l'incolore, l'impondérable et le glacé.

Quand Supervielle pense à un disparu, il ne voit pas, comme Baudelaire, un cadavre qui se décompose en puissantes exhalaisons; il assiste à l'évanouissement de sa silhouette dans le vide grandissant de la mémoire. Elle se disperse, devenant un quasi-néant impalpable: une inflexion sans visage, un soupir rêvant qu'il soupire. Ainsi Supervielle nous présente comme des images de la mort, des sillages sans barques, une brise marine qui rappelle seule l'océan, un chant d'oiseau dans une absence de forêt...

C'est à peine exister qu'être sans corps. Dieu lui-même n'est pas satisfait de sa nature purement spirituelle. S'il a donné à l'homme une enveloppe de chair opaque, de l'appétit, une voix sonore, une place dans un coin déterminé de l'univers, c'est qu'il regrette de n'avoir jamais soif, d'être invisible, silencieux, solitaire, impossible à situer.

De même, les trépassés qui ont laissé leur cadavre au creux d'une fosse, mènent une vie d'ennui, sans dangers, sans passions, sans avenir, sans bruit, sans mystère et, surtout, sans pesanteur. «Mutilés de tout leur corps», ils ne peuvent plus rien étreindre et vaquent à des semblants d'affaires dans des semblants de villes.

Plus question ici d'âmes joyeuses d'avoir rejeté leur chrysalide et de vagabonder à travers l'espace, mais des âmes en peine, des fantômes aveugles qui reviennent assiéger les vivants pour voir leurs yeux et se rachauffer à leur souffle. Le monde est plein de ces ombres frôleuses qui nous flairent, qui nous palpent, mélancoliques. Meme les arbres défunts cherchent à travers l'espace à rejoindre leurs vallées familières:

«Fantômes de peupliers

Alignés comme sur terre

Vous cherchez une rivière

Pour la longer dignement» ${ }^{48}$.

On comprend que Supervielle ait écrit sur la première page de ses Gravitations le beau vers de Tristan l'Hermite: «Lorsque nous serons morts nous parlerons de vie»».

47 Le corps tragique, París, Gallimard, 1959, p. 49.

48 «Ascension», Gravitations, p. 146. 
Les âmes «veuves», c'est-à-dire privées du corps auquel étaient liés leurs pensées et leurs souvenirs, et qu'à tort elles ont dédaigné et maudit, prennent conscience tout à coup de ce qui les lie à ce compagnon dont elles complotaient la perte ${ }^{49}$. Avec la disparition du corps, l'âme n'est que néant, que pure absence. Cet oiseau maître du ciel est coupé de ses racines, de ses sens, «ne sachant où se poser».

En fin de compte, la dialectique du corps et de l'âme n'existe pas: ils ne font qu'un. L'imagination du poète distingue l'âme du corps, ella les campe face à face pour les besoins de la fable où elle les introduit. Mais elle ne remet pas en cause leur union effective. Supervielle n'est pas plus tenté par le spiritualisme quand il chante l'âme que par le matérialisme quand il chante le corps. Il respecte, dans l'un comme dans l'autre cas, le mystère, l'ambiguïté de l'incarnation:
«Mon âme suit mon corps
La nuit comme le jour.
Elle n'a pas besoin
Du soleil pour être ombre» ${ }^{50}$.

Supervielle reste donc comme le poète qui a su parler des parties du corps, des organes, des viscères ainsi que de l'esprit de l'âme. Il ne prévoit pas un nouveau monde spiritualisé, mais bien plutôt une résurrection du corps et de l'esprit. Pour Supervielle la séparation du corps et de l'âme ne peut jamais apporter une solution heureuse à la tragique situation de l'homme.

Corps et âme prenant ainsi chacun conscience de ses limites et de sa faiblesse, se réconcilient, car l'un sans l'autre est infiniment vulnérable.

De quelque côte que l'on aborde l'oeuvre de Supervielle, c'est toujours pour voir tomber quelque vieille muraille entre les domaines que les mots séparent. Ainsi en est-il du corps et de l'amê. Or, l'oeuvre de Jules Supervielle est le chant d'amour de ce couple d'amis de toujours. C'est de l'univers, d'un univers de chair et de terre, d'arbres et de fleuves, que la fantaisie et le rêve auront besoin pour nous évoquer le mystère des régions de l'âme obscures et lointaines.

49 Dans «Disparition» (Gravitations, pp. 209-211), comme dans une enquête de police, se présente l'hypothèse du suicide (vers 27-31). L'assassin est découvert: ce n'était autre que l'âme (vers 40). Le texte de 1925 expliquait clairement les mobiles du crime: incompatibilité d'humeurs. La meurtrière y était définie comme l'alliée du squelette:

«Bête blanche et onaniste,

Tapie au meilleur de nous-mêmes

Et qui nous saute à la gorge au premier moment d'inattention».

Autrement dit, «Disparition» signifie un désaccord interne, une anxiété née du conflit de deux puissances: l'une qui est à son aise dans l'univers, qui est accordée à son rythme, c'est le corps; l'autre, sournoise, égoïstement enfermée dans les replis de la conscience, c'est l'âme. Guarnamiru (ce poème appartient à la section intitulée «Poèmes de Guanamiru») est tout entier avec la première, Supervielle déchiré entre les deux.

50 «Lâme proche», Les amis inconnus, p. 145. 
